Research article

\title{
Antipolymer antibody in Italian fibromyalgic patients
}

\author{
Laura Bazzichi', Camillo Giacomelli2, Francesca De Feo ${ }^{1}$, Tiziana Giuliano', Alessandra Rossi², \\ Marica Doveri ${ }^{1}$, Chiara Tani ${ }^{1}$, Russell B Wilson ${ }^{3}$ and Stefano Bombardieri ${ }^{1}$
}

\author{
1'Department of Internal Medicine, Division of Rheumatology, University of Pisa, Pisa, Italy \\ 2Department of Psychiatry, Neurobiology, Pharmacology and Biotechnology, University of Pisa, Pisa, Italy \\ ${ }^{3}$ Autoimmune Technologies, L.L.C. 1010 Commons Suite 1705, New Orleans, LA 70112, USA \\ Corresponding author: Laura Bazzichi, I.bazzichi@int.med.unipi.it
}

Received: 21 May 2007 Revisions requested: 10 Jul 2007 Revisions received: 27 Jul 2007 Accepted: 6 Sep 2007 Published: 6 Sep 2007

Arthritis Research \& Therapy 2007, 9:R86 (doi:10.1186/ar2285)

This article is online at: http://arthritis-research.com/content/9/5/R86

(c) 2007 Bazzichi et al.; licensee BioMed Central Ltd.

This is an Open Access article distributed under the terms of the Creative Commons Attribution License (http://creativecommons.org/licenses/by/

2.0), which permits unrestricted use, distribution, and reproduction in any medium, provided the original work is properly cited.

\section{Abstract}

The objectives of the present study were to evaluate the presence of antipolymer antibody (APA) seropositivity in 285 Italian patients affected by primary fibromyalgia (FM) and to verify whether APA levels correlate with disease severity and with cytokine levels.

APA levels were determined on serum samples by an indirect ELISA kit that detects IgG APA. Cytokines (IL-1, IL-6, IL-8, IL-10 and TNF $\alpha$ ) were measured by ELISA in plasma. The impact of FM on the quality of life was estimated using the Fibromyalgia Impact Questionnaire, while pain severity was evaluated using a visual analogic scale. Patients were also characterized by the presence of tiredness, stiffness, nonrestorative sleep, anxiety, depression, tension headache, irritable bowel syndrome, temporomandibular dysfunction and Raynaud's phenomena.
Using a cut-off value of $30 \mathrm{U}$, APA-positive values were detected in $60 \mathrm{FM}$ patients $(21.05 \%)$ and in 15 healthy control individuals $(15.00 \%)$ without significant differences among their levels or the percentage of seropositivity. FM patients with moderate and severe symptoms had slightly higher APA levels with respect to patients with mild symptoms. APA-seropositive patients exhibited significant correlations between APA levels and the Fibromyalgia Impact Questionnaire estimate $(P=$ 0.042), tiredness $(P=0.003)$ and $\mathrm{IL}-1$ levels $(P=0.0072)$.

In conclusion, APA cannot be considered a marker of disease in Italian FM patients. The presence of APA, however, might permit the identification of a subset of FM patients with more severe symptoms and of patients who may respond differently to different therapeutic strategies.

\section{Introduction}

Fibromyalgia (FM) is a syndrome defined by widespread pain for longer than 3 months and by the presence of $\geq 11$ of 18 tender points [1]. Most FM patients report fatigue, disrupted or nonrestorative sleep, mood disturbances, exercise-induced symptom flares and multiple other syndromes (for example, restless leg syndrome, irritable bowel syndrome and chronic headaches) [1-3]. Physical and emotional health as well as quality of life is often seriously impaired [4-6]. Women are the most affected (9:1 ratio of women to men affected). Like many other clinical syndromes, FM has no single specific feature but represents a complex of symptoms of self-reported or clinical deduction. Unfortunately, there is still no standardized laboratory test to detect FM or to measure its severity.
Researchers have suggested that many of the symptoms reported by women with silicone gel-filled breast implants appear to be similar to those observed in patients with FM [7]. Tenenbaum and colleagues [8] reported that many silicone breast implant recipients produced serum antibodies that recognized what initially appeared to be a high-molecular-weight antigen. After further characterization, it was determined that this antigen was not a protein, but a complex composed of partially polymerized acrylamide. Because of the polymer nature of the antigen, these antibodies have been named antipolymer antibodies (APAs).

Because of the suggested similarities between FM and reported symptoms by patients with silicone gel-filled breast implants, one of us examined the association of APA and FM and found that $47 \%$ of patients in a general rheumatological 
setting in the United States were seropositive for the presence of APA [9]. Consequently, other researchers began to investigate the APA seroactivity in patients with FM, finding controversial data $[10,11]$.

In light of these results, we examined the APA levels in a cohort of Italian FM patients and investigated their association with disease severity and cytokine levels. To determine whether APA results from a generalized autoimmune response, the APA seroreactivity was evaluated also in several autoimmune disease noncase groups including rheumatoid arthritis, Sjögren's syndrome, systemic sclerosis, systemic lupus erythematosus and undifferentiated connective-tissue disease.

\section{Materials and methods Patients}

We recruited 285 consecutive patients (270 females, 15 males) affected by primary FM as assessed by the 1990 American College of Rheumatology criteria [12], 40 noncase individuals (16 rheumatoid arthritis cases, two Sjögren's syndrome cases, 16 systemic lupus erythematosus cases, four systemic sclerosis cases, two undifferentiated connective-tissue disease cases) and 100 healthy age-matched and sex-matched subjects. Individuals with a history of silicone gelfilled breast implants or breast surgery were excluded from the study. Written consent was obtained from all participants after a full explanation of the procedure.

For each patient the tenderness at tender points was evaluated by means of the Fischer dolorimeter [13]. A rheumatologist advanced the instrument at a rate of $4 \mathrm{~kg} / \mathrm{s}$ and the patient was instructed to say when this procedure became painful. The pain threshold was calculated from 18 points, and the tender point count was determined by the number of tender points that had a threshold $\leq 4 \mathrm{~kg} / \mathrm{cm}^{2}$. The total fibromyalgic tender point score (right + left) was used in the statistical analysis. Each positive tender point had a pain score between 0 and 3. The Tender Point Index was calculated as the sum of each positive tender point score divided by the total number of tender points. The total pain severity and tiredness were evaluated by a visual analogical scale (0-10).

To estimate the impact of FM on the quality of life, all patients and control individuals received a Fibromyalgia Impact Questionnaire $[14,15]$ consisting of 10 items. The total score ranged from 0 (no impact) to 100 (maximum impact).

All patients were asked whether they had frequently suffered any of the following symptoms [16] in the past 12 months: tiredness, sleep disturbance, anxiety, depression, irritable bowel syndrome, constipation, diarrhoea, Raynaud's phenomenon, paresthesiae, articular stiffness, muscular stiffness, dry eyes, dry mouth, temporomandibular disorders, tension headache, allergy, low back pain, restless leg syndrome, gastro- esophageal reflux disease, burning/pain with urination, dizziness, allodynia, traumatic event, blurred vision and sore throat.

We arbitrarily classified the FM severity on the basis of the tender point count: the presence of 11 tender points was considered mild severity, the presence of 14-16 tender points as moderate and the presence of more than 16 tender points was designated severe.

\section{Antipolymer antibody and interleukin assay}

APA levels were determined on serum samples by an indirect ELISA kit that detects IgG APA (Corgenix, Westminster, CO, USA), according to the manufacturer's instructions. Diluted serum samples, calibrator and control samples were incubated in polymer-coated microwells. After the removal of unbound proteins by washing, specific antibodies for human IgG labelled with horseradish peroxidase were added, forming complexes with the polymer-bound antibodies. The bound enzyme-antibody conjugate was assayed by the addition of a single solution containing tetramethylbenzidine and hydrogen peroxide as the chromogenic substrate. Colour develops in the wells at an intensity proportional to the concentration of APA. Results were obtained by reading the optical density at $450 \mathrm{~nm}$. The measure of the optical density was converted in the sample unit using a conversion factor. All the samples were tested in duplicate, and a result of more $30 \mathrm{U}$ was considered positive, as suggested by the manufacturer.

Cytokines (IL-1, IL-6, IL-8, IL-10 and TNF $\alpha$ ) were measured by ELISA in plasma (Bender MedSystem, Austria, Vienna). A polyclonal antibody-coating antibody is adsorbed onto microwells, and the cytokine present in the samples or standards binds to antibodies adsorbed to the microwells. A biotin-conjugated monoclonal antibody directed to cytokine is added and binds to cytokine captured by the first antibody. Streptavidin-horseradish peroxidase is added and binds to the biotinconjugated cytokine. Unbound streptavidin-horseradish peroxidase is removed during a washing step, and substrate solution reactive with horseradish peroxidase is added to the wells. A coloured product is formed in proportion to the amount of cytokine present in the sample. The reaction is terminated by addition of acid and the absorbance is measured at $450 \mathrm{~nm}$. A standard curve is prepared from seven cytokine standard dilutions and the cytokine sample concentration is determined.

\section{Statistical analysis}

Data were analysed by means of nonparametric statistical methods using Kruskal-Wallis analysis of variance, Spearman's correlation, Student's $t$ test and the chi-square test. $P<$ 0.05 was considered statistically significant.

\section{Results}

Demographic and clinical data of the $285 \mathrm{FM}$ patients are presented in Table 1. There are no significant differences 
Table 1

Demographic characteristics, clinical characteristics and antipolymer antibody (APA) levels of 285 fibromyalgia patients.

\begin{tabular}{llll}
\hline & All fibromyalgia patients & Patients with APA $>30 U$ & Patients with APA <30 U \\
\hline Gender (females/males) & $270 / 15$ & $57 / 3$ & $213 / 12$ \\
Age (years) (median (range)) & $54(19-82)$ & $46(19-74)$ & $56(20-82)$ \\
Tender points & $14.29 \pm 0.29$ & $13.88 \pm 0.83$ & $14.30 \pm 0.34$ \\
Tender Point Index & $2.17 \pm 0.04$ & $2.17 \pm 0.12$ & $2.19 \pm 0.04$ \\
Onset disease & $9.19 \pm 0.60$ & $8.77 \pm 1.19$ & $10.11 \pm 0.67$ \\
Fibromyalgia Impact & $58.21 \pm 1.22$ & $55.89 \pm 2.59$ & $58.46 \pm 1.39$ \\
Questionnaire & $6.96 \pm 0.17$ & $7.16 \pm 0.41$ & $6.88 \pm 0.19$ \\
Tiredness & $6.80 \pm 0.17$ & $6.31 \pm 0.42$ & $6.87 \pm 0.18$ \\
Pain &
\end{tabular}

Results expressed as the mean \pm standard error of the mean. The Tender Point Index was calculated as the sum of each positive tender point score divided by the total number of tender points.

between the FM subset with positive APA and those FM patients with negative APA. The serum APA level (mean \pm standard error of the mean) was $22.45 \pm 2.55 \mathrm{U}$ in the FM patients and was $19.93 \pm 3.74 \mathrm{U}$ in the control individuals (Figure 1).

Considering a cut-off value of $30 \mathrm{U}$, APA-positive values were detected in $60 \mathrm{FM}$ patients $(21.05 \%)$ and in 15 healthy control individuals (15.00\%) without significant differences in seropositivity among them.

Patients with moderate $(19.17 \pm 4.44)$ and severe symptoms $(25.05 \pm 4.42)$ had slightly higher APA levels with respect to patients with mild symptoms (13.18 \pm 2.01$)$ (Figure 1). No correlations were found between the APA levels of FM patients and the onset of the disease, the tender point counts or scores, or pain.

A negative correlation between APA levels and age was found both in FM patients $(P<0.0001)$ and in control individuals $(P$ $=0.0294$ ).

Considering the subset of FM patients with positive APA, significant correlations were found between APA levels versus the Fibromyalgia Impact Questionnaire $(P=0.042)$ and between APA levels versus tiredness $(P=0.003)$.

\section{Figure 1}

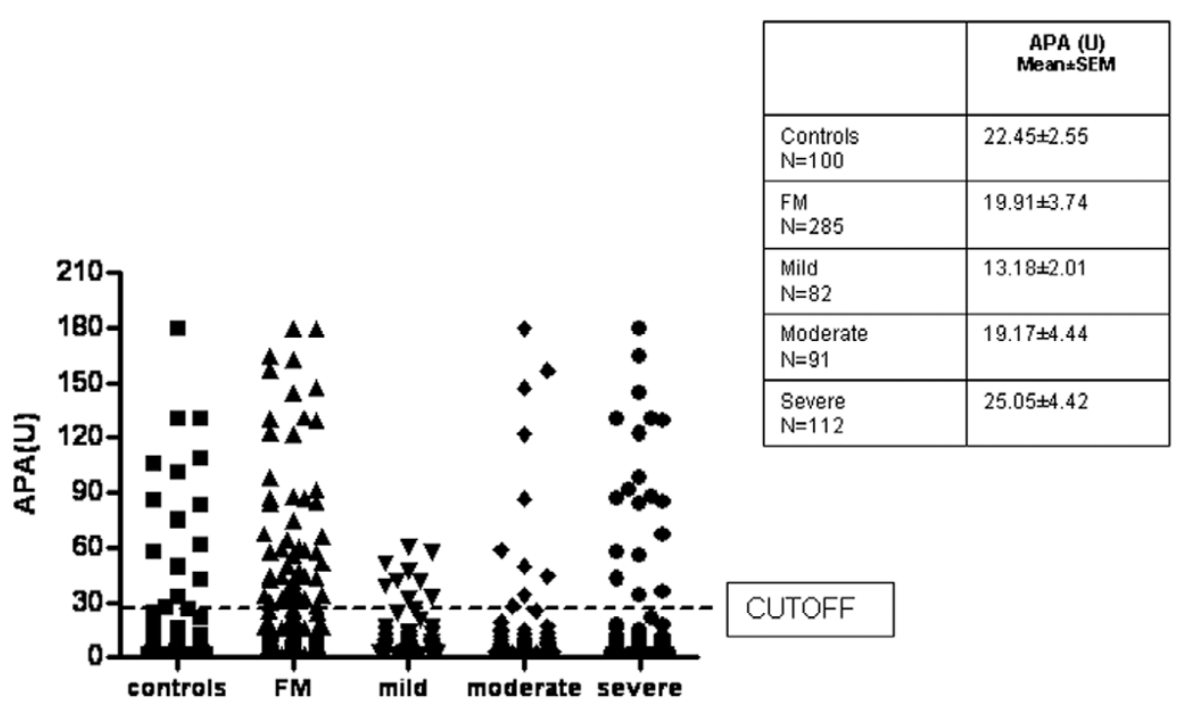

Antipolymer antibody levels in fibromyalgia patients and in control individuals. Antipolymer antibody (APA) levels in fibromyalgia (FM) patients (all patients and patients separated according to disease severity) and in control individuals. SEM, standard error of the mean. 
Figure 2

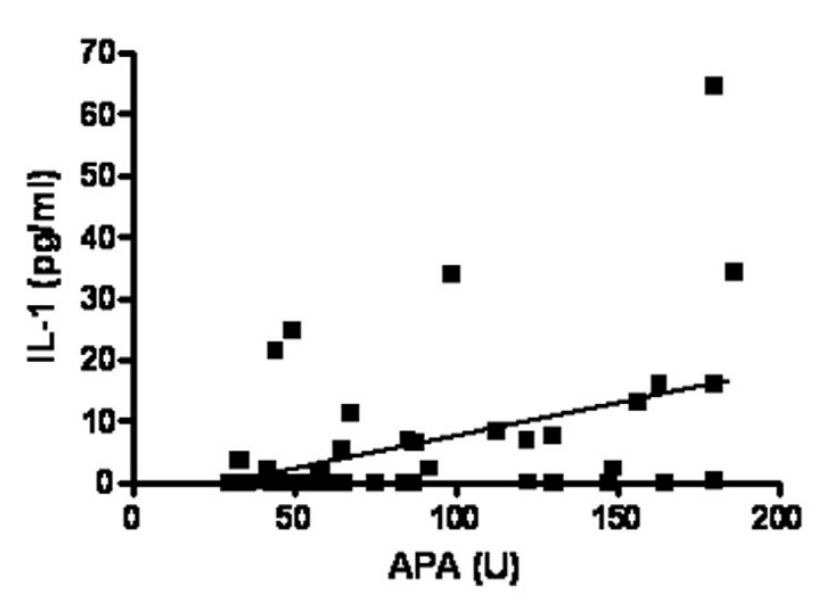

Correlation between antipolymer antibody and IL-1 levels. Correlation between antipolymer antibody (APA) and IL-1 levels in the subgroup of fibromyalgia patients with APA $>30 \mathrm{U}$.

Patients with positive APA levels showed a lower percentage of constipation $(26 \%$ versus $42 \%, P<0.05)$ and a higher percentage of sore throat $(43.5 \%$ versus $24.4 \%, P<0.05)$.

No differences in cytokine levels were detected between FM patients with mild, moderate or severe symptoms or between those FM patients with APA-positive/negative values. A significant correlation was found, however, between APA levels and IL-1 values within the subset of patients with positive APA ( $P$ $=0.0072$; Figure 2).

APA seroreactivity results were low (7.7\%) in the autoimmune noncase group.

\section{Discussion}

The production of APA antibodies may result from an immunological response to self-antigens or to environmental agents, such as medications, silicone, or other chemicals, which results in the formation of antibodies cross-reacting with partially polymerized acrylamide. Because it has been shown that APA antibodies are present in about $50 \%$ of patients with silicone breast implants, and the highest prevalence of APA is found in implant patients with severe symptoms of a FM-like syndrome [8], subjects with a history of silicone gel-filled breast implants or breast surgery were excluded from the present study.

Our objective was to examine the presence of APA seropositivity in 285 nonimplanted FM patients and to verify whether APA levels correlate with disease severity and with cytokine levels.

We did not find differences of seropositivity between FM patients and control individuals but did observe that patients with moderate and severe symptoms exhibited qualitatively higher levels of APA, even if not statistically significant, with respect to patients with mild symptoms. Lee and colleagues [11] also did not find differences between Korean FM patients and control individuals, but reported a lower percentage of seropositivity (7.2\%). Wilson and colleagues [9], on the contrary, studying an American population, found a higher percentage of seropositivity in FM patients (47\%) while the percentage reported for control individuals was similar to ours.

Wilson and colleagues [9], like the present study, observed higher APA levels with the presence of more severe symptoms, while other authors [11] showed a downward trend as the symptom severity increased.

Interestingly, in our study the APA levels correlated with Fibromyalgia Impact Questionnaire estimates and tiredness results in the subset of seropositive patients, so APA correlated with the severity of the disease only when it is positive. This is the first study reporting an association between APA seropositivity and the Fibromyalgia Impact Questionnaire.

Like other reports $[10,11]$, we found that the APA assay was negatively associated with age, probably because APA is not associated with autoimmune diseases and with a T-helper-2 response. We found a positive correlation between APA and IL-1 levels in the patient subgroup with APA $>30 \mathrm{U}$, which might be associated with an immunological response to environmental agents in some way related to polymerized acrylamide. The higher percentage of sore throat in the seropositive patients might in some way be linked to this response. This possible association is further strengthened by previous observations of elevated levels of APA in patients with infection-associated malfunctions of silicone-based ventriculoperitoneal shunts [17].

We found a low prevalence of APA seroactivity in the noncase group that is about one-half of that found in FM patients. This result is in accordance with a precedent work [8], re-emphasizing the thesis that APA is not a general marker for autoimmune disease process.

\section{Conclusion}

We have shown that APA cannot be considered a marker of disease in Italian FM patients. Its presence, however, might permit the identification of a subset of FM patients with more severe symptoms and who may respond differently to different therapeutic strategies. APA seroactivity is also not a general marker for autoimmune disease processes.

\section{Competing interests}

The authors declare that they have no competing interests.

\section{Authors' contributions}

LB conceived of the study, its design and coordination, evaluated the clinical parameters and helped to draft the manu- 
script. CG, FDF and TG performed the laboratory assays. AR performed the statistical analysis and drafted the manuscript. MD and CT participated in the evaluation of clinical parameters. RBW provided the kit and helped to draft the manuscript. SB participated in the design of the study. All authors read and approved the final manuscript.

\section{Acknowledgements}

The authors are grateful to Dr Wendy Doherty for assistance in the preparation of the manuscript and to Mrs Marisa Rasi for her nursing.

\section{References}

1. Bennett R: Fibromyalgia: present to future. Curr Rheumatol Rep 2005, 7:371-376.

2. Clauw DJ, Crofford LJ: Chronic widespread pain and fibromyalgia: what we know and what we need to know. Best Pract Res Clin Rheumatol 2003, 17:685-701.

3. Matsutani LA, Marques AP, Ferreira EA, Assumpção A, Lage LV, Casarotto RA, Pereira CA: Effectiveness of muscle stretching exercises with and without laser therapy at tender points for patients with fibromyalgia. Clin Exp Rheumatol 2007, 25:410-415.

4. Burckhardt CS, Clark SR, Bennett RM: Fibromyalgia and quality of life: a comparative analysis. J Rheumatol 1993, 20:475-479.

5. Strombeck B, Ekdahl C, Manthorpe R, Wikstrom I, Jacobsson L: Health-related quality of life in primary Sjogren's syndrome, rheumatoid arthritis and fibromyalgia compared to normal population data using SF-36. Scand J Rheumatol 2000, 29:20-28

6. Bazzichi L, Rossi A, Massimetti G, Giannaccini G, Giuliano T, De Feo F, Ciapparelli A, Dell'Osso L, Bombardieri S: Cytokine patterns in fibromyalgia and their correlation with clinical manifestations. Clin Exp Rheumatol 2007, 25:225-230.

7. Wolfe $F$, Anderson J: Silicone filled breast implants and the risk of fibromyalgia and rheumatoid arthritis. J Rheumatol 1999, 26:2025-2028.

8. Tenenbaum SA, Rice JC, Espinoza LR, Cuellar ML, Plymale DR, Sander DM, Williamson LL, Haislip AM, Gluck OS, Tesser JRP, et al.: Use of antipolymer antibody assay in recipients of silicone breast implants. Lancet 1997, 349:449-454.

9. Wilson RB, Gluck OS, Tesser JR, Rice JC, Meyer A, Bridges AJ: Antipolymer antibody reactivity in a subset of patients with fibromyalgia correlates with severity. J Rheumatol 1999, 26:402-407.

10. Jensen $B$, Wittrup IH, Wiik A, Bliddal H, Friis AS, McLaughlin JK, Danneskiold-Samsoe B, Olsen $\mathrm{JH}$ : Antipolymer antibodies in Danish fibromyalgia patients. Clin Exp Rheumatol 2004, 22:227-229.

11. Lee SS, Yoon HJ, Park YW: Antipolymer antibody is not associated with fibromyalgia in Korean female patients. Rheumatol Int 2006, 27:73-77.

12. Wolfe F, Smythe HA, Yunus MB, Bennett RM, Bombardier C, Goldenberg DL, Tugwell P, Campbell SM, Abeles M, Clark P, et al.: The American College of Rheumatology 1990 Criteria for the Classification of Fibromyalgia. Report of the Multicenter Criteria Committee. Arthritis Rheum 1990, 33:160-172.

13. Fisher AA: Pressure tolerance over muscles and bones in normal subjects. Arch Phys Med Rehabil 1986, 67:406-409.

14. Sarzi-Puttini P, Atzeni F, Fiorini T, Panni B, Randisi G, Turiel M, Carabba M: Validation of an Italian version of the Fibromyalgia Impact Questionnaire (FIQ-I). Clin Exp Rheumatol 2003, 21:459-464.

15. Bennett R: The Fibromyalgia Impact Questionnaire (FIQ): a review of its development, current version, operating characteristics and uses. Clin Exp Rheumatol 2005, 23:154-162.

16. ARA Glossary Committee: Dictionary of the Rheumatic Diseases. Vol 1: Signs and Symptoms New York: Contact Press; 1982.

17. Vandevord PJ, Gupta N, Wilson RB, Vinuya RZ, Schaefer CJ, Canady Al, Wooley PH: Immune reactions associated with silicone-based ventriculo-peritoneal shunt malfunctions in children. Biomaterials 2004, 25:3853-3860. 